September 2006

\title{
Flavor Symmetry and Vacuum Aligned Mass Textures
}

\author{
Satoru Kaneko ${ }^{1}$, Hideyuki Sawanaka ${ }^{2}$, Takaya Shingai ${ }^{2}$, \\ Morimitsu Tanimoto ${ }^{3}$ and Koichi Yoshioka ${ }^{4}$ \\ ${ }^{1}$ Physics Department, Ochanomizu University, Tokyo 112-8610, Japan \\ ${ }^{2}$ Graduate School of Science and Technology, Niigata University, Niigata 950-2181, Japan \\ ${ }^{3}$ Physics Department, Niigata University, Niigata 950-2181, Japan \\ ${ }^{4}$ Physics Department, Kyushu University, Fukuoka 812-8581, Japan
}

\begin{abstract}
The mass matrix forms of quarks and leptons are discussed in theory with permutation flavor symmetry. The structure of scalar potential is analyzed in case that electroweak doublet Higgs fields have non-trivial flavor symmetry charges. We find that realistic forms of mass matrices are obtained dynamically in the vacuum of the theory, where some of Higgs bosons have vanishing expectation values which lead to vanishing elements in quark and lepton mass matrices. Mass textures are realized in the true vacuum and their positions are controlled by flavor symmetry. An interesting point is that, due to the flavor group structure, the up and down quark mass matrices are automatically made different in the vacuum, which lead to non-vanishing generation mixing. It is also discussed that flavor symmetry is needed to be broken in order not to have too light scalars. The lower bounds of Higgs masses are derived from the experimental data of flavor-changing rare processes such as the neutral $\mathrm{K}$ meson mixing.
\end{abstract}




\section{Introduction}

Flavor symmetry is expected to be a clue to understand the masses and mixing angles of quarks and leptons. It reduces the number of free parameters in Yukawa couplings, and some testable predictions of masses and mixing angles generally follow. The discrete non-Abelian

symmetries contain the symmetry groups $S_{n}$, the dihedral groups $D_{n}$, the binary dihedral (quaternion) groups $Q_{n}$ and the tetrahedral groups $A_{n}$. Some predictive models with discrete flavor symmetries have been explored by many authors [1]-8].

The simplest discrete non-Abelian group is $S_{3}$. The Higgs sector has a rich structure in theory with the $S_{3}$ flavor symmetry, e.g. there are two possibilities that $S U(2)$ doublet Higgs fields have trivial and non-trivial flavor symmetry charges. The former case has been discussed intensively in the literature (see for example Ref. [2]). The latter case is also expected to have characteristic phenomenology where Yukawa couplings of quarks and leptons are described by renormalizable operators.

In this paper, we discuss the mass matrix forms of quarks and leptons in the case that $S U(2)$ doublet Higgs fields have non-trivial $S_{3}$ flavor charges. We perform the analysis of Higgs potential at the electroweak scale and examine whether some of Higgs bosons have vanishing expectation values. In this case, vanishing elements (texture zeros) of fermion mass matrices are obtained dynamically in the vacuum of the theory, and their positions are controlled by flavor symmetry. Such zeros in the mass matrices have often been assumed by hand [9]-[17], unlike our scheme. An interesting point of the scheme is that, due to the $S_{3}$ group structure, the up and down quark mass matrices automatically have different forms at the vacuum, which lead to non-vanishing generation mixing. The suppression of flavor-changing neutral currents (FCNC) mediated by multiple Higgs fields will also be discussed.

This paper is organized as follows. In Section 2, we present some fundamentals of the $S_{3}$ group and the $S_{3}$ symmetry invariant form of Yukawa couplings (mass matrices) in the supersymmetric case. In Sections 3 and 4, the symmetry-invariant Higgs potential is constructed and its structure is analyzed, especially focusing on the conditions for vanishing vacuum expec- 


\begin{tabular}{c|ccc} 
& $\mathbf{1}_{\mathrm{S}}$ & $\mathbf{1}_{\mathrm{A}}$ & 2 \\
\hline $\mathbf{1}_{\mathrm{S}}$ & $\mathbf{1}_{\mathrm{S}}$ & $\mathbf{1}_{\mathrm{A}}$ & 2 \\
$\mathbf{1}_{\mathrm{A}}$ & $\mathbf{1}_{\mathrm{A}}$ & $\mathbf{1}_{\mathrm{S}}$ & 2 \\
2 & 2 & 2 & $2+\mathbf{1}_{\mathrm{A}}+\mathbf{1}_{\mathrm{S}}$
\end{tabular}

Table 1: $S_{3}$ tensor products.

tation values (VEVs). In Section 5, we show that fermion mass matrices favored by the recent experimental data are highly limited in our $S_{3}$ framework. In Sections 6 and 7, several other phenomenological issues, i.e. the Higgs spectrum with flavor symmetry breaking effects and tree-level FCNC processes, are investigated. Section 8 is devoted to summarizing our results.

\section{$2 S_{3}$ invariant mass matrix}

In this section, the $S_{3}$-invariant forms of mass matrices are presented. For more details of the $S_{3}$ group structure and symmetry-invariant forms, see, for example, Ref. [2]. The $S_{3}$ group has three irreducible representations; two singlets and one doublet, which we denote throughout this paper as $\mathbf{1}_{\mathbf{S}}$ (singlet), $\mathbf{1}_{\mathbf{A}}$ (pseudo singlet) and $\mathbf{2}$ (doublet), respectively. The decomposition of tensor products is shown in Table 1. A non-trivial product is $\mathbf{2} \times \mathbf{2}=\mathbf{2}+\mathbf{1}_{\mathbf{A}}+\mathbf{1}_{\mathbf{S}}$. For two doublets $\psi=\left(\psi_{1}, \psi_{2}\right)^{T}$ and $\phi=\left(\phi_{1}, \phi_{2}\right)^{T}$, that is explicitly given by

$$
\psi \times \phi=\left(\begin{array}{c}
\psi_{1}^{\dagger} \phi_{2} \\
\psi_{2}^{\dagger} \phi_{1}
\end{array}\right)_{2}+\left(\psi_{1}^{\dagger} \phi_{1}-\psi_{2}^{\dagger} \phi_{2}\right)_{\mathbf{1}_{\mathbf{A}}}+\left(\psi_{1}^{\dagger} \phi_{1}+\psi_{2}^{\dagger} \phi_{2}\right)_{\mathbf{1}_{\mathbf{S}}},
$$

where the suffixes in the right hand side of the equation denote $S_{3}$ representations. Another form of product is written by using $\psi_{C} \equiv\left(\psi_{2}^{*}, \psi_{1}^{*}\right)^{T}$ which behaves as a doublet. The tensor product of $\psi_{C}$ with another doublet $\phi$ becomes

$$
\psi_{C} \times \phi=\left(\begin{array}{c}
\psi_{2} \phi_{2} \\
\psi_{1} \phi_{1}
\end{array}\right)_{\mathbf{2}}+\left(\psi_{1} \phi_{2}-\psi_{2} \phi_{1}\right)_{\mathbf{1}_{\mathbf{A}}}+\left(\psi_{1} \phi_{2}+\psi_{2} \phi_{1}\right)_{\mathbf{1}_{\mathbf{S}}}
$$

Since this latter form of product does not contain any complex conjugates, it is useful for describing, e.g. Majorana masses for neutrinos and holomorphic terms in supersymmetric theory. It is mentioned that the two types of $S_{3}$ invariants given above correspond to two invariant tensors of $S U(2)$, which contains $S_{3}$ as a subgroup. 
Let us discuss quark/lepton mass matrices, where both left-handed and right-handed fermions transform under a single $S_{3}$ symmetry. We suppose that two of three generations belong to $S_{3}$ doublets and the others are singlets. In this paper, we consider supersymmetric theory and then matter superfields of first two generations $\Psi_{L_{1,2}}$ and $\Psi_{R_{1,2}}$ are treated as $S_{3}$ doublets, into which left and right-handed fermions are embedded as $\psi_{L_{1,2}}$ and $\psi_{R_{1,2}}^{c}$, respectively. As for $S U(2)$ weak doublet Higgses, all three types of $S_{3}$ irreducible representations are introduced; a doublet $\left(H_{1}, H_{2}\right)^{T}$, a pseudo singlet $H_{A}$ and a singlet $H_{S}$ for each of up and down type Higgs sector. It is found from the tensor product (2.2) that the most general supersymmetric Yukawa (mass) terms are written as

$$
\begin{gathered}
W_{y}=\Psi_{L i}\left(M_{D}\right)_{i j} \Psi_{R j}, \\
M_{D}=\left(\begin{array}{cc|c}
a H_{1} & b H_{S}+c H_{A} & d H_{2} \\
b H_{S}-c H_{A} & a H_{2} & d H_{1} \\
\hline e H_{2} & e H_{1} & f H_{S}
\end{array}\right),
\end{gathered}
$$

where $a, b, \cdots, f$ are independent Yukawa coupling constants.

It is noticed that, if some of Higgs bosons have vanishing expectation values in the vacuum of the theory, corresponding mass matrix elements vanish and then mass (not Yukawa) texture is realized. That is the scheme we adopt in this paper for quark/lepton mass matrices. A nontrivial issue is whether such VEVs are obtained in the electroweak symmetry breaking vacuum. Therefore the Higgs potential should be carefully examined.

Finally, the $S_{3}$-invariant bare Majorana mass for matter superfield $\Psi_{i}(i=1,2,3)$ is given by

$$
\begin{gathered}
W_{m}=\Psi_{i}\left(M_{R}\right)_{i j} \Psi_{j}, \\
M_{R}=\left(\begin{array}{ll|l}
M_{1} M_{1} & \\
\hline & & M_{2}
\end{array}\right) .
\end{gathered}
$$




\section{$3 \quad S_{3}$ Higgs scalar potential}

Let us consider supersymmetric theory with $S_{3}$ flavor symmetry and introduce the following Higgs superfields:



The symmetry-invariant supersymmetric Lagrangian is

$$
\begin{gathered}
\mathcal{L}=\int d^{2} \theta d^{2} \bar{\theta} K+\left(\int d^{2} \theta W+\text { h.c. }\right) \\
K=\sum_{\alpha=S, A, 1,2}\left(\hat{H}_{u \alpha}^{\dagger} e^{G_{u}} \hat{H}_{u \alpha}+\hat{H}_{d \alpha}^{\dagger} e^{G_{d}} \hat{H}_{d \alpha}\right), \\
W=\mu_{S} \hat{H}_{u S} \epsilon \hat{H}_{d S}+\mu_{A} \hat{H}_{u A} \epsilon \hat{H}_{d A}+\mu_{D}\left(\hat{H}_{u 1} \epsilon \hat{H}_{d 2}+\hat{H}_{u 2} \epsilon \hat{H}_{d 1}\right),
\end{gathered}
$$

where $G_{u, d} \equiv \pm \frac{1}{2} g_{Y} \hat{V}_{Y}+g_{2} \hat{V}_{2}$ with the electroweak vector superfields $\hat{V}_{Y}$ and $\hat{V}_{2}$, and $g_{Y}$ and $g_{2}$ are the corresponding gauge coupling constants. The mass parameters $\mu_{S, A, D}$ are generally complex and $\epsilon$ is the antisymmetric tensor for $S U(2)$ weak indices $\left(\epsilon^{12}=1\right)$. The Higgs scalar potential is then given by

$$
\begin{gathered}
V=V_{\text {susy }}+V_{\text {soft }}, \\
V_{\text {susy }}=\frac{1}{2} D_{Y}^{2}+\frac{1}{2} \sum_{a=1,2,3}\left(D^{a}\right)^{2} \\
+\left|\mu_{S}\right|^{2}\left(H_{u S}^{\dagger} H_{u S}+H_{d S}^{\dagger} H_{d S}\right)+\left|\mu_{A}\right|^{2}\left(H_{u A}^{\dagger} H_{u A}+H_{d A}^{\dagger} H_{d A}\right) \\
+\left|\mu_{D}\right|^{2}\left(H_{u 1}^{\dagger} H_{u 1}+H_{d 1}^{\dagger} H_{d 1}+H_{u 2}^{\dagger} H_{u 2}+H_{d 2}^{\dagger} H_{d 2}\right) \\
V_{\text {soft }}= \\
\quad m_{u S}^{2} H_{u S}^{\dagger} H_{u S}+m_{d S}^{2} H_{d S}^{\dagger} H_{d S}+\left(b_{S} H_{u S} \epsilon H_{d S}+\text { h.c. }\right) \\
+m_{u A}^{2} H_{u A}^{\dagger} H_{u A}+m_{d A}^{2} H_{d A}^{\dagger} H_{d A}+\left(b_{A} H_{u A} \epsilon H_{d A}+\text { h.c. }\right) \\
+m_{u D}^{2}\left(H_{u 1}^{\dagger} H_{u 1}+H_{u 2}^{\dagger} H_{u 2}\right)+m_{d D}^{2}\left(H_{d 1}^{\dagger} H_{d 1}+H_{d 2}^{\dagger} H_{d 2}\right) \\
+\left[b_{D}\left(H_{u 1} \epsilon H_{d 2}+H_{u 2} \epsilon H_{d 1}\right)+\text { h.c. }\right]
\end{gathered}
$$


where $b_{x}, m_{u x}$ and $m_{d x}(x=S, A, D)$ are the holomorphic and non-holomorphic mass parameters of supersymmetry breaking, respectively. The $D$ terms are explicitly given by

$$
\begin{aligned}
D_{Y}= & -\frac{1}{2} g_{Y}\left(H_{u S}^{\dagger} H_{u S}-H_{d S}^{\dagger} H_{d S}+H_{u A}^{\dagger} H_{u A}-H_{d A}^{\dagger} H_{d A}\right. \\
& \left.+H_{u 1}^{\dagger} H_{u 1}-H_{d 1}^{\dagger} H_{d 1}+H_{u 2}^{\dagger} H_{u 2}-H_{d 2}^{\dagger} H_{d 2}\right) \\
D^{a}= & -g_{2}\left(H_{u S}^{\dagger} T^{a} H_{u S}+H_{d S}^{\dagger} T^{a} H_{d S}+H_{u A}^{\dagger} T^{a} H_{u A}+H_{d A}^{\dagger} T^{a} H_{d A}\right. \\
& \left.+H_{u 1}^{\dagger} T^{a} H_{u 1}+H_{d 1}^{\dagger} T^{a} H_{d 1}+H_{u 2}^{\dagger} T^{a} H_{u 2}+H_{d 2}^{\dagger} T^{a} H_{d 2}\right)
\end{aligned}
$$

with $T^{a}(a=1,2,3)$ is the $S U(2)$ weak generators. It should be noted that $V_{\text {soft }}$ is introduced as the most general supersymmetry-breaking Higgs soft terms compatible with the $S_{3}$ symmetry.

We here mention a possibility of spontaneous breakdown of CP symmetry. The following terms in $V_{\text {soft }}$ which contain complex couplings could be sources of $\mathrm{CP}$ violation:

$$
b_{S} H_{u S} \epsilon H_{d S}+b_{A} H_{u A} \epsilon H_{d A}+b_{D}\left(H_{u 1} \epsilon H_{d 2}+H_{u 2} \epsilon H_{d 1}\right)+\text { h.c. } .
$$

Analyzing the stationary conditions for the Higgs potential, it is understood that the phases of Higgs VEVs should satisfy $\theta_{u \alpha}+\theta_{d \alpha}=0(\alpha=S, A, 1,2)$ where $\theta_{u \alpha(d \alpha)}$ are the up(down)-type Higgs phases. While these phases do not directly appear in the Higgs scalar potential at the vacuum, they cannot be removed in general by field redefinitions unlike in the minimal supersymmetric standard model (MSSM). Therefore there is a possibility of causing spontaneous CP breakdown by taking account of complex phases of Higgs VEVs in Higgs-mediated processes.

\section{Analysis of Higgs scalar potential}

Hereafter we assume that the charged Higgs fields do not develop nonzero VEVs so as to make the $U(1)_{E M}$ symmetry remains intact after the electroweak symmetry breaking. The $S_{3}$ Higgs 
scalar potential we analyze thus becomes

$$
\begin{aligned}
V= & \left(\left|\mu_{S}\right|^{2}+m_{u S}^{2}\right) v_{u S}^{2}+\left(\left|\mu_{S}\right|^{2}+m_{d S}^{2}\right) v_{d S}^{2}-2 b_{S} v_{u S} v_{d S} \\
& +\left(\left|\mu_{A}\right|^{2}+m_{u A}^{2}\right) v_{u A}^{2}+\left(\left|\mu_{A}\right|^{2}+m_{d A}^{2}\right) v_{d A}^{2}-2 b_{A} v_{u A} v_{d A} \\
& +\left(\left|\mu_{D}\right|^{2}+m_{u D}^{2}\right)\left(v_{u 1}^{2}+v_{u 2}^{2}\right)+\left(\left|\mu_{D}\right|^{2}+m_{d D}^{2}\right)\left(v_{d 1}^{2}+v_{d 2}^{2}\right) \\
& -2 b_{D}\left(v_{u 1} v_{d 2}+v_{u 2} v_{d 1}\right)+\frac{g_{Y}^{2}+g_{2}^{2}}{8} X^{2} \\
& X \equiv v_{u S}^{2}-v_{d S}^{2}+v_{u A}^{2}-v_{d A}^{2}+v_{u 1}^{2}-v_{d 1}^{2}+v_{u 2}^{2}-v_{d 2}^{2},
\end{aligned}
$$

where $v_{x}$ are the absolute values of Higgs scalars:

$$
\begin{aligned}
& v_{u S}=\left|\left\langle H_{u S}^{0}\right\rangle\right|, \quad v_{u A}=\left|\left\langle H_{u A}^{0}\right\rangle\right|, \quad v_{u 1}=\left|\left\langle H_{u 1}^{0}\right\rangle\right|, \quad v_{u 2}=\left|\left\langle H_{u 2}^{0}\right\rangle\right|, \\
& v_{d S}=\left|\left\langle H_{d S}^{0}\right\rangle\right|, \quad v_{d A}=\left|\left\langle H_{d A}^{0}\right\rangle\right|, \quad v_{d 1}=\left|\left\langle H_{d 1}^{0}\right\rangle\right|, \quad v_{d 2}=\left|\left\langle H_{d 2}^{0}\right\rangle\right|,
\end{aligned}
$$

and the parameters $b_{S, A, D}$ have been chosen to be real positive by field redefinitions. Notice that the scalar potential (4.1) is invariant under the label-exchanging transformations, $1 \leftrightarrow 2$, $S \leftrightarrow A$ and/or $u \leftrightarrow d$.

First we discuss the instability of scalar potential at the origin of field space. The potential parameters have to satisfy at least one of the following conditions in order to break the electroweak gauge symmetry:

$$
\begin{aligned}
b_{S}^{2} & >\left(\left|\mu_{S}\right|^{2}+m_{u S}^{2}\right)\left(\left|\mu_{S}\right|^{2}+m_{d S}^{2}\right), \\
b_{A}^{2} & >\left(\left|\mu_{A}\right|^{2}+m_{u A}^{2}\right)\left(\left|\mu_{A}\right|^{2}+m_{d A}^{2}\right), \\
b_{D}^{2} & >\left(\left|\mu_{D}\right|^{2}+m_{u D}^{2}\right)\left(\left|\mu_{D}\right|^{2}+m_{d D}^{2}\right) .
\end{aligned}
$$

Then the equations of motion become

$$
\begin{aligned}
& \left(\left|\mu_{S}\right|^{2}+m_{u S}^{2}\right) v_{u S}=b_{S} v_{d S}-X^{\prime} v_{u S}, \quad\left(\left|\mu_{S}\right|^{2}+m_{d S}^{2}\right) v_{d S}=b_{S} v_{u S}+X^{\prime} v_{d S} \\
& \left(\left|\mu_{A}\right|^{2}+m_{u A}^{2}\right) v_{u A}=b_{A} v_{d A}-X^{\prime} v_{u A}, \quad\left(\left|\mu_{A}\right|^{2}+m_{d A}^{2}\right) v_{d A}=b_{A} v_{u A}+X^{\prime} v_{d A} \\
& \left(\left|\mu_{D}\right|^{2}+m_{u D}^{2}\right) v_{u 1}=b_{D} v_{d 2}-X^{\prime} v_{u 1}, \quad\left(\left|\mu_{D}\right|^{2}+m_{d D}^{2}\right) v_{d 2}=b_{D} v_{u 1}+X^{\prime} v_{d 2} \\
& \left(\left|\mu_{D}\right|^{2}+m_{u D}^{2}\right) v_{u 2}=b_{D} v_{d 1}-X^{\prime} v_{u 2}, \quad\left(\left|\mu_{D}\right|^{2}+m_{d D}^{2}\right) v_{d 1}=b_{D} v_{u 2}+A^{\prime} v_{d 1}
\end{aligned}
$$


where $X^{\prime}=\frac{g_{Y}^{2}+g_{2}^{2}}{4} X$. These equations (4.7)-(4.10) depend respectively on $v_{u S(d S)}, v_{u A(d A)}, v_{u 1(d 2)}$ and $v_{u 2(d 1)}$, except in the $X^{\prime}$ parts. The $Z$ boson mass is given by

$$
m_{Z}^{2}=\frac{1}{2}\left(g_{Y}^{2}+g_{2}^{2}\right)\left(v_{u S}^{2}+v_{d S}^{2}+v_{u A}^{2}+v_{d A}^{2}+v_{u 1}^{2}+v_{d 1}^{2}+v_{u 2}^{2}+v_{d 2}^{2}\right)
$$

In addition, substituting (4.7)-(4.10) for the scalar potential, we find the depth of the potential at the minimum:

$$
V_{\min }=-\frac{g_{Y}^{2}+g_{2}^{2}}{8} X^{2} \leq 0
$$

This expression also holds in the case of $S_{3}$ symmetry breaking model discussed in later section.

Next we study the possibility of having vanishing VEVs in the minimum of the potential. In general, (4.7)-(4.10) are the coupled equations through the $X$ parts. We separate these equations into three parts for the singlet (4.7), the pseudo singlet (4.8), and the doublet (4.9) and (4.10). As for the singlet and pseudo singlet parts, each coefficients of $X^{\prime}$ in (4.7) and (4.8) are $v_{u(d) S}$ and $v_{u(d) A}$, respectively. Therefore vanishing VEVs makes the equations trivial within each sector. There is however a bit difference in the $S_{3}$ doublet part [Eqs. (4.9) and (4.10)], that is, they cannot be separated from each other. These equations have not only a relation through the $X$ part but also a common parameter $b_{D}$ which originates from the symmetry invariance.

\section{1 $S_{3}$ singlet Higgses}

First let us examine the vanishing VEVs of $S_{3}$ singlet Higgs fields. In this case, we take the other Higgs VEVs arbitrary. The analysis is the same for pseudo singlet Higgs fields. There are two possible patterns of zero VEVs; one is that both of the up and down-type Higgs VEVs are zero and another is that only one of them is zero.

- $v_{u S}=v_{d S}=0$

This solution always exists. Though such a solution does not make sense in models with one pair of Higgs doublets like the MSSM, it is possible in the present case as long as some of instability conditions for other Higgs parts are satisfied. 
- $v_{u S}=0, v_{d S} \neq 0$ or $\quad v_{d S}=0, v_{u S} \neq 0$

It is found that the parameter condition $b_{S}=0$ is necessary for this solution. For example, if one supposes $v_{u S}=0$ and $v_{d S} \neq 0$, the stationary conditions mean

$$
b_{S}=0 \quad \text { and } \quad\left|\mu_{S}\right|^{2}+m_{d S}^{2}-\frac{g_{Y}^{2}+g_{2}^{2}}{4}\left(-v_{d S}^{2}+X_{0}\right)=0
$$

where we have defined $X_{0}=\left.X\right|_{v_{u S}=v_{d S}=0}$. Therefore as long as the parameter $b_{S}$ is nonzero, the solution with one of VEVs being zero does not exist. It is noted that the condition $b_{S}=0$ is difficult to be satisfied exactly at any scale, and hence such a type of solution may not be realistic.

\section{$4.2 \quad S_{3}$ doublet Higgses}

Including $S_{3}$ doublet Higgs fields makes the theory complex but is promising. Unlike the case of $S_{3}$ singlet Higgses, all the four VEVs, $v_{u 1}, v_{u 2}, v_{d 1}$ and $v_{d 2}$ should be simultaneously taken into account because $b_{D}$ is a common parameter as we mentioned above. In the analysis, we take the singlet Higgs VEVs, $v_{u S}, v_{d S}, v_{u A}$ and $v_{d A}$, to be arbitrary. It is important to notice that the stationary conditions for $S_{3}$ doublet Higgses are described (except in the $X$ parts) by two pairs of VEVs: Eq. (4.9) for $\left(v_{u 1}, v_{d 2}\right)$ and Eq. (4.10) for $\left(v_{u 2}, v_{d 1}\right)$. It can be seen from the result in the previous section that the existence of VEV pairs means that the VEV forms such as $v_{u 1}=0$ and $v_{d 2} \neq 0$ are not allowed, i.e. only one VEV in each pair does not develop a nonzero VEV unless an unlikely condition $b_{D}=0$ is satisfied.

There are $16\left(=2^{4}\right)$ patterns for the $4 \mathrm{VEVs}$ of $S_{3}$ doublets. Among them, only 7 patterns are theoretically independent due to the label-exchanging invariances of the potential: $1 \leftrightarrow 2$ and $u \leftrightarrow d$, mentioned before. For example, in the case that $v_{u 1}=v_{d 2}=0$ and $v_{u 2}, v_{d 1} \neq 0$, the equations of motion give

$$
2\left|\mu_{D}\right|^{2}+m_{u D}^{2}+m_{d D}^{2}=b_{D}\left(\frac{v_{u 2}}{v_{d 1}}+\frac{v_{d 1}}{v_{u 2}}\right) .
$$

A similar form of vacuum equation is obtained for a theoretically equivalent case that $v_{u 2}=$ $v_{d 1}=0$ and $v_{u 1}, v_{d 2} \neq 0$ by use of label exchanges. Therefore it is enough to consider either 


\begin{tabular}{|c|c|c|c|c|}
\hline$\overline{v_{u 1}}$ & $v_{u 2}$ & $v_{d 1}$ & $v_{d 2}$ & conditions \\
\hline 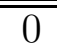 & $\overline{0}$ & 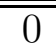 & $\overline{0}$ & \\
\hline 0 & 0 & $\varnothing$ & 0 & $b_{D}=0$ \\
\hline 0 & $\varnothing$ & $\varnothing$ & $\overline{0}$ & \\
\hline 0 & 0 & $\varnothing$ & $\varnothing$ & $b_{D}=0$ \\
\hline 0 & $\varnothing$ & 0 & $\varnothing$ & $b_{D}=0, \quad 2\left|\mu_{D}\right|^{2}+m_{u D}^{2}+m_{d D}^{2}=0$ \\
\hline$\varnothing$ & $\varnothing$ & 0 & $\bar{\varnothing}$ & $b_{D}=0, \quad 2\left|\mu_{D}\right|^{2}+m_{u D}^{2}+m_{d D}^{2}=0$ \\
\hline$\varnothing$ & $\varnothing$ & $\varnothing$ & $\varnothing$ & \\
\hline
\end{tabular}

Table 2: All representative VEV patterns of the $S_{3}$ doublet Higgs fields. The blank entries denote no needs of parameter conditions.

\begin{tabular}{|c|c|c|c|}
\hline$v_{u 1}$ & $v_{u 2}$ & $v_{d 1}$ & $v_{d 2}$ \\
\hline \hline 0 & 0 & 0 & 0 \\
\hline 0 & $\varnothing$ & $\varnothing$ & 0 \\
\hline$\varnothing$ & 0 & 0 & $\varnothing$ \\
\hline$\varnothing$ & $\varnothing$ & $\varnothing$ & $\varnothing$ \\
\hline
\end{tabular}

Table 3: All VEV patterns of the $S_{3}$ doublet Higgs fields without parameter conditions.

of these patterns. All 7 possible patterns obtained from the potential analysis are shown in Table 2, It is noted that the parameter conditions given in Table 2, i.e. $b_{D}=0$ and $2\left|\mu_{D}\right|^{2}+m_{u D}^{2}+m_{d D}^{2}=0$, are unlikely to be satisfied exactly, because these relations are not protected by symmetry arguments and generally unstable against quantum corrections (though there are some models for a vanishing $b$ parameter at a given scale [18]). Therefore in this paper we do not consider the solutions with non-trivial parameter conditions. In the end, we have found the relevant vacuum solutions without parameter conditions, which are presented in Table 3 ,

To summarize the results in Section 4, we have found all possible minima of the scalar potential for $S_{3}$ singlet and doublet Higgs fields (Table 4). These solutions do not require any exact tuning of Lagrangian parameters for electroweak symmetry breaking and are physically available. The potential depth at each minimum is controlled by Higgs mass parameters in the Lagrangian, and any of the VEV patterns in Table 4 can be made the global vacuum of the theory, as seen in Eq. (4.12). 


\begin{tabular}{|c|c|c|c|c|c|c|c|}
\hline$v_{u S}$ & $v_{d S}$ & $v_{u A}$ & $v_{d A}$ & $v_{u 1}$ & $v_{u 2}$ & $v_{d 1}$ & $v_{d 2}$ \\
\hline \hline 0 & 0 & 0 & 0 & 0 & & & 0 \\
\hline 0 & 0 & 0 & 0 & & 0 & 0 & \\
\hline 0 & 0 & 0 & 0 & & & & \\
\hline 0 & 0 & & & 0 & 0 & 0 & 0 \\
\hline 0 & 0 & & & 0 & & & 0 \\
\hline 0 & 0 & & & & 0 & 0 & \\
\hline 0 & 0 & & & & & & \\
\hline & & 0 & 0 & 0 & 0 & 0 & 0 \\
\hline & & 0 & 0 & 0 & & & 0 \\
\hline & & 0 & 0 & & 0 & 0 & \\
\hline & & 0 & 0 & & & & \\
\hline & & & & 0 & 0 & 0 & 0 \\
\hline & & & & 0 & & & 0 \\
\hline & & & & & 0 & 0 & \\
\hline & & & & & & & \\
\hline
\end{tabular}

Table 4: All possible minima of the scalar potential for $S_{3}$ singlet and doublet Higgs fields without tuning of Lagrangian parameters for electroweak symmetry breaking. The blank entries denote non-vanishing VEVs.

\section{$5 \quad$ Quark and lepton mass textures}

As a phenomenological application of the vacuum analysis performed in the previous section, we study in this section the mass matrix forms of quarks and leptons derived from $S_{3}$ flavor symmetry. Let us consider a supersymmetric theory with three-generation quark and lepton superfields in addition to the Higgs content previously analyzed. The three generations belong to a reducible 3 representation of $S_{3}$, that is, two of three generations behave as a doublet and the other is a singlet or a pseudo singlet. When the first two generation superfields consist of $S_{3}$ doublets, the most general form of mass matrix is given by (2.4) with non-vanishing Higgs VEVs.

A usual approach often seen in the literature to obtain realistic forms of mass matrices is to control coupling constants of effective Yukawa operators with additional symmetries or to adjust coupling constants by hand so that the experimental data is reproduced. Unlike these approaches, our strategy in this paper is to dynamically realize mass matrix forms (mass 
textures) in the vacuum of the theory. That is, as discussed in the previous section, some of Higgs VEVs vanish at the minimum of scalar potential, which in turn lead to texture (zero) forms of quark/lepton mass matrices. The available candidates of vacua are listed in Table 4. One can see from the table that the VEV structures of up and down-type Higgs fields are not parallel and rather different due to the group properties of $S_{3}$ flavor symmetry. This fact is favorable in light of the experimental data of fermion masses and mixing angles, which data is well known to show that the up and down quark sectors would have highly different generation structures.

In the following, we assume as an example that the $S_{3}$ representations of three-generation matter fields are $\mathbf{2}+\mathbf{1}_{\mathbf{S}}$. If one adopts $\mathbf{1}_{\mathbf{A}}$ instead of $\mathbf{1}_{\mathbf{S}}$, phenomenological results are changed according to Eq. (2.2). Examining the vacuum patterns in Table 4, we find that the following case with four zero VEVs leads to the simplest texture (i.e. the maximal number of zero matrix elements) with non-trivial flavor mixing:

$$
v_{u 1}=v_{d 2}=v_{u S}=v_{d S}=0, \quad v_{u 2}, v_{d 1}, v_{u A}, v_{d A} \neq 0
$$

The VEV pattern obtained by interchanging the labels $1 \leftrightarrow 2$ is also the case. That can be covered by exhausting the generation label exchanges of matter fields, and therefore we safely focus on the vacuum (5.1) and examine all types of $S_{3}$ charge assignments of matter fields. In what follows, we use the notation that $Q_{i}, u_{i}, d_{i}, L_{i}, e_{i}$, and $\nu_{i}(i=1,2,3)$ are the superfields of left-handed quarks, right-handed up quarks, right-handed down quarks, left-handed leptons, right-handed charged leptons and right-handed neutrinos, respectively.

\subsection{Quark mass textures}

When the first and second generation quarks behave as $S_{3}$ doublets, the up and down quark mass textures in the vacuum (5.1) are read from the generic form of mass matrix (2.4):

$$
M_{u}=\left(\begin{array}{ccc} 
& \beta v_{u A} & \gamma v_{u 2} \\
-\beta v_{u A} & \alpha v_{u 2} & \\
\delta v_{u 2} & &
\end{array}\right), \quad M_{d}=\left(\begin{array}{ccc}
\bar{\alpha} v_{d 1} & \bar{\beta} v_{d A} \\
-\bar{\beta} v_{d A} & & \bar{\gamma} v_{d 1} \\
& \bar{\delta} v_{d 1} &
\end{array}\right)
$$


where the blank entries mean zeros, and $\alpha, \beta, \cdots, \bar{\gamma}$ and $\bar{\delta}$ are the Yukawa coupling constants. In this flavor basis, the above matrices appear not to lead to appropriate mass hierarchies and generation mixing. We have exhausted the $S_{3}$ charge assignments of matter fields and found that only the following 4 cases are almost consistent with the current experimental data of fermion masses and mixing angles.

\subsection{1 $\left(\mathrm{Q}_{2}, \mathrm{Q}_{1}\right)+\mathrm{Q}_{3}, \quad\left(\mathrm{u}_{3}, \mathrm{u}_{2}\right)+\mathrm{u}_{1}, \quad\left(\mathrm{~d}_{2}, \mathrm{~d}_{3}\right)+\mathrm{d}_{1}$}

The title of this subsection means the $S_{3}$ charge assignment that the second and first generations of left-handed quark superfields consist of a doublet and the third one $Q_{3}$ is a singlet, and similarly for $u_{i}$ and $d_{i}$. In this case, the quark mass matrices are given by

$$
M_{u}=\left(\begin{array}{ccc} 
& b_{u} & -c_{u} \\
d_{u} & c_{u} & \\
& & i_{u}
\end{array}\right), \quad M_{d}=\left(\begin{array}{ccc}
a_{d} & -b_{d} & \\
& e_{d} & b_{d} \\
& & i_{d}
\end{array}\right)
$$

In the first order approximation, they predict the quark mass eigenvalues and mixing angles

$$
\begin{array}{rcc}
m_{u}=-\frac{b_{u} d_{u}}{c_{u}}, & m_{c}=c_{u}, & m_{t}=i_{u}, \\
m_{d}=a_{d}, & m_{s}=e_{d}, & m_{b}=i_{d}, \\
V_{u s}=-\frac{b_{u}}{c_{u}}-\frac{b_{d}}{e_{d}}, & V_{c b}=\frac{b_{d}}{i_{d}}, & V_{u b}=\frac{c_{u}}{i_{u}}-\frac{b_{u} b_{d}}{c_{u} i_{d}},
\end{array}
$$

which lead to a relation among the observables:

$$
V_{u b}-\frac{m_{c}}{m_{t}}=V_{c b}\left(V_{u s}+\frac{m_{b}}{m_{s}} V_{c b}\right)
$$

Such parameter-independent relation may be useful to examine whether the model can well describe the observations. If one evaluates the relation (5.7) with respect to the mixing matrix element $V_{c b}$, one third of the observed value is reproduced, which implies a necessity of some modification of the mass matrix forms. 


\subsection{2 $\left(\mathrm{Q}_{3}, \mathrm{Q}_{2}\right)+\mathrm{Q}_{1}, \quad\left(\mathrm{u}_{1}, \mathrm{u}_{2}\right)+\mathrm{u}_{3}, \quad\left(\mathrm{~d}_{3}, \mathrm{~d}_{2}\right)+\mathrm{d}_{1}$}

In the second case, $\left(Q_{3}, Q_{2}\right),\left(u_{1}, u_{2}\right)$ and $\left(d_{3}, d_{2}\right)$ are the $S_{3}$ doublets. The mass matrices then take the forms:

$$
M_{u}=\left(\begin{array}{ccc}
a_{u} & & \\
-d_{u} & e_{u} & \\
& d_{u} & i_{u}
\end{array}\right), \quad M_{d}=\left(\begin{array}{ccc} 
& b_{d} & \\
d_{d} & & -f_{d} \\
& f_{d} & i_{d}
\end{array}\right) .
$$

The flavor charge assignments of $Q_{i}$ and $d_{i}$ are the same, which leads to a generation-symmetric texture form like the Fritzsch ansatz [9] for the down quark mass matrix. Also such charge assignment might be relevant to the flipped $S U(5)$ unified models [19]. The prediction for the quark mass eigenvalues and mixing angles is given by

$$
\begin{array}{ccc}
m_{u}=\frac{a_{u} e_{u}}{\sqrt{d_{u}^{2}+e_{u}^{2}}}, & m_{c}=\sqrt{d_{u}^{2}+e_{u}^{2}}, & m_{t}=i_{u}, \\
m_{d}=-\frac{b_{d} d_{d} i_{d}}{f_{d}^{2}}, & m_{s}=\frac{f_{d}^{2}}{i_{d}}, & m_{b}=i_{d}, \\
V_{u s}=\frac{b_{d} i_{d}}{f_{d}^{2}}, & V_{c b}=-\frac{f_{d}}{i_{d}}, & V_{u b}=\frac{b_{d} f_{d}}{i_{d}^{2}},
\end{array}
$$

in the first order approximation. It is noted that the leading contributions to the mixing angles mainly come from the down quark sector, and then satisfy the following two relations among the observables:

$$
\left|V_{u b}\right|=\left|V_{u s}\right|\left(\frac{m_{s}}{m_{b}}\right)^{3 / 2}, \quad\left|V_{c b}\right|=\sqrt{\frac{m_{s}}{m_{b}}} .
$$

These do not so largely deviate from the experimental data, but predict a bit large (small) value for the mixing matrix element $V_{c b}$ (for the mass eigenvalue $m_{s}$ ).

\subsection{3 $\left(\mathrm{Q}_{2}, \mathrm{Q}_{3}\right)+\mathrm{Q}_{1}, \quad\left(\mathrm{u}_{2}, \mathrm{u}_{3}\right)+\mathrm{u}_{1}, \quad\left(\mathrm{~d}_{2}, \mathrm{~d}_{1}\right)+\mathrm{d}_{3}$}

The third pattern is the case that $\left(Q_{2}, Q_{3}\right),\left(u_{2}, u_{3}\right)$ and $\left(d_{2}, d_{1}\right)$ are the $S_{3}$ doublets, which lead to the mass textures:

$$
M_{u}=\left(\begin{array}{ccc} 
& b_{u} & \\
d_{u} & & f_{u} \\
& -f_{u} & i_{u}
\end{array}\right), \quad M_{d}=\left(\begin{array}{ccc}
a_{d} & & \\
d_{d} & e_{d} & \\
& -d_{d} & i_{d}
\end{array}\right) .
$$


This type of charge assignment is compatible with $S U(5)$ grand unification [20]. The masses and mixing angles are determined in the first order as

$$
\begin{array}{ccc}
m_{u}=-\frac{b_{u} d_{u} i_{u}}{f_{u}^{2}}, & m_{c}=\frac{f_{u}^{2}}{i_{u}}, & m_{t}=i_{u}, \\
m_{d}=\frac{a_{d} e_{d}}{\sqrt{d_{d}^{2}+e_{d}^{2}}}, & m_{s}=\sqrt{d_{d}^{2}+e_{d}^{2}}, & m_{b}=i_{d}, \\
V_{u s}=-\frac{b_{u} i_{u}}{f_{u}^{2}}, & V_{c b}=-\frac{f_{u}}{i_{u}}, & V_{u b}=\frac{b_{u}}{f_{u}} .
\end{array}
$$

It is noted that the leading contributions to the mixing angles mainly come from the up quark sector, and then satisfy the following two relations among the observables:

$$
\left|V_{c b}\right|=\sqrt{\frac{m_{c}}{m_{t}}}, \quad\left|V_{u s} V_{c b}\right|=\left|V_{u b}\right|
$$

with which we would have some discrepancy between the prediction and the experimental data.

\subsection{4 $\left(\mathrm{Q}_{2}, \mathrm{Q}_{3}\right)+\mathrm{Q}_{1}, \quad\left(\mathrm{u}_{2}, \mathrm{u}_{3}\right)+\mathrm{u}_{1}, \quad\left(\mathrm{~d}_{1}, \mathrm{~d}_{2}\right)+\mathrm{d}_{3}$}

The last case is the $S_{3}$ charges; $\left(Q_{2}, Q_{3}\right),\left(u_{2}, u_{3}\right)$ and $\left(d_{1}, d_{2}\right)$ are the doublets, which assignment is also consistent with $S U(5)$ unification. Note that this type of assignment differs from the third case above, only for the doublet constitution of right-handed down quarks in the first and second generations. Therefore the third and this fourth cases are physically equivalent if all parameter spaces were taken into account. That is, these two cases correspond to two separate parameter regions in a single theory, both of which regions are phenomenologically viable. The mass texture forms now become

$$
M_{u}=\left(\begin{array}{ccc} 
& b_{u} & \\
d_{u} & & f_{u} \\
& -f_{u} & i_{u}
\end{array}\right), \quad M_{d}=\left(\begin{array}{ccc} 
& b_{d} & \\
d_{d} & e_{d} & \\
-e_{d} & & i_{d}
\end{array}\right),
$$

which give the masses and mixing angles in the first order approximation:

$$
\begin{array}{ccc}
m_{u}=-\frac{b_{u} d_{u} i_{u}}{f_{u}^{2}}, & m_{c}=\frac{f_{u}^{2}}{i_{u}}, & m_{t}=i_{u}, \\
m_{d}=-\frac{b_{d} d_{d}}{e_{d}}, & m_{s}=e_{d}, & m_{b}=i_{d}, \\
V_{u s}=-\frac{b_{u} i_{u}}{f_{u}^{2}}+\frac{b_{d}}{e_{d}}, & V_{c b}=-\frac{f_{u}}{i_{u}}, & V_{u b}=\frac{b_{u}}{f_{u}} .
\end{array}
$$


There are 8 free parameters for 9 observables, and we have one predictive relation

$$
\left|V_{c b}\right|=\sqrt{\frac{m_{c}}{m_{t}}}
$$

This relation is known to well fit the experimentally observed values and has already been discussed in other theoretical frameworks [16, 21]. However the precise numerical estimation indicates that the equation (5.22) is not exactly satisfied with the observational data: $\left|V_{c b}\right|=0.039-0.044$ and $\sqrt{m_{c} / m_{t}}=0.057-0.064$ [22]. Some remedies can easily be found. The discrepancy is removed with radiative corrections, for instance, the renormalization-group effects on masses and mixing angles. A naive and probable source of such effects is the large top-quark Yukawa coupling. (The strong gauge coupling does not affect mass ratios and generation mixing due to its flavor universality.) For example, in the MSSM we obtain the renormalization-group equation

$$
\frac{d \ln \left(\left|V_{c b}\right| / \sqrt{m_{c} / m_{t}}\right)}{d \ln \mu}=\frac{1}{32 \pi^{2}}\left(y_{t}^{2}-y_{b}^{2}\right) .
$$

where $\mu$ is the renormalization scale and $y_{t}, y_{b}$ are the top and bottom Yukawa couplings, respectively. The positive coefficient of the top-Yukawa contribution implies that the discrepancy is indeed reduced in lower energy regime. It is however noted that in the present model the flavor symmetry is supposed to be broken at low energy such as the electroweak scale and hence the scale dependence might not be enough to make the relation (5.22) fulfilled.

Another possible source of radiative corrections comes from supersymmetry-breaking parameters. The scalar fermions propagate in the loops and the chirality is flipped via holomorphic or non-holomorphic couplings of scalar quarks. In both of these cases, the corrections do not change zero structure of mass textures but could modify nonzero matrix elements, depending on supersymmetry-breaking mass parameters of scalar quarks. For example, if the $\left(M_{u}\right)_{23,32}$ elements receive such corrections, the relation (5.22) is modified and the theory would become viable in light of the current experimental data. We leave a detailed study of model dependence on supersymmetry-breaking parameters of matter fields to future investigations. 
In the present analysis, we have neglected CP-violating phases. All the mass matrix elements in this subsection can be taken to be real by quark phase redefinitions. This fact implies that the mass matrices are diagonalized by real orthogonal matrices up to overall phase rotations. In this case the observable $\mathrm{CP}$ phase is induced by the presence of different phase rotations between the up and down left-handed quarks. This is the case for the quark mass matrices in this subsection. The prediction of quark sector $\mathrm{CP}$ violation is consistent with the experimental data as long as the mixing angles are properly reproduced.

\subsection{Lepton mass textures}

Under the standard model gauge symmetry, the flavor charge assignment and Yukawa couplings of the lepton sector are free from those of the quark sector. One could explore the patterns of $S_{3}$ charges for lepton fields, just as in the previous analysis for the quark sector, so that various phenomenological constraints are satisfied. However an attractive way to determine lepton flavor charges is to promote the theory to be embedded into grand unification, where lepton and quark multiplets are unified and have the same flavor charges. Along this line of thought, the cases 2,3 , and 4 in the previous section are the candidates to be considered. It is however easily found that the cases 2 and 3 are not reconciled with the experimental data of lepton sector, even if one includes additional Higgs fields in higher-dimensional representations of unified gauge symmetry. Thus we find the unique solution, the case 4 (in Section 5.1.4), for $S_{3}$ flavor charges of lepton fields which would be compatible both with unification hypothesis and the observed data. It may be interesting to remind that, only from the analysis of quark sector performed in the previous sections, the case 4 has been phenomenologically singled out.

Let us first consider the charged lepton sector. We assume here $S U(5)$ grand unification because the quark flavor charges in the case 4 are consistent with it. The left-handed charged leptons $L_{i}$ are combined with the right-handed down quarks into unified gauge multiplets (anti quintuplets), and therefore $\left(L_{1}, L_{2}\right)$ consists of an $S_{3}$ doublet and $L_{3}$ a singlet. Similarly, $\left(e_{2}, e_{3}\right)$ transforms as an $S_{3}$ doublet and $e_{1}$ as a singlet. The charged lepton mass matrix $M_{e}$ is given 
by the transpose of that of down quarks $M_{d}$ in (5.18) and becomes at low energy

$$
M_{e} \simeq r\left(\begin{array}{ccc}
d_{d} & 3 e_{d} \\
b_{d} & -3 e_{d} & \\
& & i_{d}
\end{array}\right)
$$

where we have included a group-theoretical factor ' -3 ' [23] in front of the element $e_{d}$. Such a factor originates from a Yukawa coupling to higher-dimensional Higgs field [e.g. 45-plet of $S U(5)] 1$ and is known to make the mass eigenvalues of charged leptons well fitted to the observed values if one takes account of renormalization-group effects on the down quark Yukawa couplings from the strong $S U(3)$ gauge sector [that has been effectively included as the factor $r$ in (5.24)]. The mass matrices $M_{d}(\underline{5.18})$ and $M_{e}(\underline{5.24})$ are found to satisfy the relations

$$
\frac{3 m_{e}}{m_{d}}=\frac{m_{\mu}}{3 m_{s}}=\frac{m_{\tau}}{m_{b}}
$$

which lead to a better explanation for the mass eigenvalues than that without the grouptheoretical factor. We have found that all the other patterns to include factors -3 are not consistent with the observation. Therefore the charged lepton mass texture (5.24) is the unique simplest solution with our vacuum aligned scheme in $S U(5)$ grand unification with $S_{3}$ flavor symmetry.

The mixing matrix which rotates the left-handed charged leptons to diagonalize $M_{e}$ is

$$
U_{e}=\left(\begin{array}{ccc}
1 & \frac{d_{d}}{3 e_{d}} & \frac{3 e_{d}}{i_{d}} \\
-\frac{d_{d}}{3 e_{d}} & 1 & -\frac{d_{d}}{i_{d}} \\
-\frac{3 e_{d}}{i_{d}} & 0 & 1
\end{array}\right)
$$

in the first order approximation. It is found from this expression that $\left|\left(U_{e}\right)_{13}\right|=m_{\mu} / m_{\tau} \simeq 1 / 17$ and $\left|\left(U_{e}\right)_{23}\right|=\left|\left(U_{e}\right)_{12}\left(U_{e}\right)_{13}\right| \ll m_{\mu} / m_{\tau}$. In particular, the latter means that the observed large lepton mixing between the second and third generations must come from the neutrino sector. As for $\left(U_{e}\right)_{12}$, a naive upper bound is obtained from the experimental upper bound on the 1-3 lepton mixing $V_{e 3} ; \quad\left|\left(U_{e}\right)_{12}\right|<\sqrt{2}\left(V_{e 3}\right)_{\max }$, where $V_{\alpha i}(\alpha=e, \mu, \tau$ and $i=1,2,3)$ is the observable lepton mixing matrix [24]. Interestingly, a lower bound on the charged lepton

\footnotetext{
${ }^{1}$ The representation of Higgs fields under unified gauge symmetry is independent of the potential analysis at the electroweak scale given in the previous section.
} 
contribution to the 1-2 lepton mixing $\left|\left(U_{e}\right)_{12}\right|$ can be expressed in terms of the observables by imagining that there should not be any fine tuning of parameters in reproducing $V_{u s}$ in (5.21). That gives $\left|\left(U_{e}\right)_{12}\right|>3\left(m_{e} / m_{\mu}\right) / V_{u s} \sim 1 / 16$.

We introduce the three generation right-handed neutrinos and utilize the seesaw mechanism [25] to obtain tiny neutrino masses. The neutrino Dirac mass texture $M_{\nu}$ in the vacuum of theory and the right-handed neutrino bare Majorana mass matrix $M_{R}$ are respectively read from the flavor symmetry invariance and given by

$$
M_{\nu}=\left(\begin{array}{ccc} 
& b_{\nu} & c_{\nu} \\
-b_{\nu} & e_{\nu} & \\
g_{\nu} & &
\end{array}\right), \quad M_{R}=\left(\begin{array}{ccc}
M_{1} & M_{1} & \\
& & M_{2}
\end{array}\right) .
$$

Here we have taken the $S_{3}$ charge of right-handed neutrinos as $\left(\nu_{1}, \nu_{2}\right)+\nu_{3}$, that is, the first two generations make a doublet. It is however noticed that the charge assignment of $\nu_{i}$ (equivalently the label changing effect of $\nu_{i}$ ) is completely irrelevant to low-energy physics and the generation structure of light neutrino mass matrix is determined only by the flavor charge of left-handed leptons $L_{i}$. After integrating out heavy modes, the light neutrino mass matrix $M_{L}=-M_{\nu} M_{R}^{-1} M_{\nu}^{T}$ becomes

$$
\begin{gathered}
M_{L}=\frac{b_{\nu}^{2}}{M_{1}}\left(\begin{array}{ccc}
-z & 1 & -x \\
1 & 2 y & -x y \\
-x & -x y & 0
\end{array}\right), \\
x=\frac{g_{\nu}}{b_{\nu}}, \quad y=\frac{e_{\nu}}{b_{\nu}}, \quad z=\frac{c_{\nu}^{2}}{b_{\nu}^{2}} \frac{M_{1}}{M_{2}} .
\end{gathered}
$$

Taking into account that the charged lepton sector has small generation mixing, it is found that this form of neutrino mass matrix is suitable for large generation mixing with the inverted mass hierarchy of light neutrinos: $m_{2} \simeq m_{1} \gg m_{3}$. The 2-3 large lepton mixing is controlled by $x \sim \mathcal{O}(1)$. The observed mass squared differences imply the traceless condition $2 y \simeq z$ $(|y|,|z| \ll 1)$. In the limit $y, z \rightarrow 0$, the $1-2$ and $2-3$ neutrino generation mixings are maximal $\left(\theta_{12}^{\nu}=\theta_{23}^{\nu}=\pi / 4\right)$ and the 1-3 angle becomes zero $\left(\theta_{13}^{\nu}=0\right)$. Including finite effects of $y$ and $z$, we obtain the first order expressions:

$$
\tan ^{2} \theta_{12}^{\nu}=1-\frac{2 y+z}{\sqrt{1+x^{2}}}, \quad \tan \theta_{23}^{\nu}=x, \quad \tan \theta_{13}^{\nu}=\frac{-x y}{\sqrt{1+x^{2}}}
$$




$$
m_{1}=\frac{b_{\nu}^{2}}{M_{2}}\left(\sqrt{1+x^{2}}-y+\frac{z}{2}\right), \quad m_{2}=\frac{b_{\nu}^{2}}{M_{2}}\left(\sqrt{1+x^{2}}+y-\frac{z}{2}\right), \quad m_{3}=0 .
$$

If the small mixing contributions from $M_{e}$ are neglected, there is a prediction which relates the observables in neutrino oscillation experiments:

$$
\frac{2 \tan \theta_{13}^{\nu}}{\tan \theta_{23}^{\nu}}+\tan \theta_{12}^{\nu}-1=\frac{m_{2}^{2}-m_{1}^{2}}{4\left(m_{2}^{2}-m_{3}^{2}\right)} .
$$

This is certainly consistent with the current experimental data. Finally, including the charged lepton contribution (5.26), we obtain

$$
\begin{aligned}
& V_{e 2}=\sin \theta_{12}^{\nu}-\left(U_{e}\right)_{12} \cos \theta_{12}^{\nu} \cos \theta_{23}^{\nu}+\left(U_{e}\right)_{13} \cos \theta_{12}^{\nu} \sin \theta_{23}^{\nu}, \\
& V_{\mu 3}=\sin \theta_{23}^{\nu}, \\
& V_{e 3}=\sin \theta_{13}^{\nu}-\left(U_{e}\right)_{12} \sin \theta_{23}^{\nu}-\left(U_{e}\right)_{13} \cos \theta_{23}^{\nu},
\end{aligned}
$$

where $\theta_{13}^{\nu} \ll 1$ has been used. It is found that $V_{e 3}$ has a lower bound; $\left|V_{e 3}\right| \geq 0.04$, which is

derived by taking account of the experimental data, $V_{e 2}, V_{\mu 3}, \Delta m_{12}^{2}$ and $\Delta m_{23}^{2}$ [22] with the relation (5.32) and the constraints on $\left(U_{e}\right)_{12,13}$ discussed above. That will be tested in future experiments such as the double Chooz [26].

In Section 5, we have shown that our scheme for generating zero texture forms with vacuum alignment is applied to mass matrices of quarks and leptons. Exhausting the patterns of matter flavor charges, we have found the highly limited numbers of charge assignments are phenomenologically viable. In particular, our scheme is also consistent with $S U(5)$ grand unification, and the uniquely determined matter flavor charges predict typical low-energy phenomenology such as the relations among the observables, independently of model parameters.

\section{$6 \quad$ Higgs mass spectrum and $S_{3}$ breaking terms}

The analysis in the previous section has shown that the vacuum (5.1) is phenomenologically interesting if applied to the quark and lepton mass textures. However the model has a problem that some massless bosons generally appear at any vacuum where more than one sets of Higgs 


\begin{tabular}{|l|cccccccc|}
\hline & $H_{u S}$ & $H_{d S}$ & $H_{u A}$ & $H_{d A}$ & $H_{u 1}$ & $H_{u 2}$ & $H_{d 1}$ & $H_{d 2}$ \\
\hline$U(1)_{X}$ & +1 & -1 & 0 & 0 & +1 & 0 & 0 & -1 \\
\hline$U(1)_{Y}$ & +1 & -1 & +1 & -1 & +1 & +1 & -1 & -1 \\
\hline
\end{tabular}

Table 5: The $U(1)$ symmetries of the Higgs scalar potential with the $S_{3}$ breaking terms (6.1).

bosons have non-vanishing VEVs. This general feature comes from the fact that the Higgs scalar potential given in Section 3 has an enhanced global symmetry $S U(2) \times U(1)^{2}$ and leads to massless Nambu-Goldstone bosons in the electroweak broken phases. It is therefore reasonable to (softly) break the flavor symmetry within the scalar potential so as not to enhance the global symmetry. In this paper we introduce supersymmetry-breaking soft terms which violate $S_{3}$ flavor symmetry. We find that the following simple set of breaking terms is appropriate for the present purpose:

$$
V_{\not_{3}}=b_{S D} H_{u S} H_{d 2}+b_{S D}^{\prime} H_{u 1} H_{d S}+b_{A D} H_{u A} H_{d 1}+b_{A D}^{\prime} H_{u 2} H_{d A}+\text { h.c. }
$$

There are several reasons why these terms are chosen: (i) The global symmetry of total Higgs potential is broken down to $U(1)_{X}$ under which $H_{u S}$ and $H_{u 1}\left(H_{d S}\right.$ and $\left.H_{d 2}\right)$ are positively (negatively) charged. Note that, combined with the $U(1)_{Y}$ hypercharge symmetry, $U(1)_{X}$ is converted to another global $U(1)$ where $H_{u A}$ and $H_{d 1}\left(H_{d A}\right.$ and $\left.H_{u 2}\right)$ are positively (negatively) charged (see Table 5). As discussed in Section 4.2, there are four sets of up and down type Higgs fields in the original potential without flavor-breaking terms, which potential therefore has four $U(1)$ invariances. Each set of $\left(H_{u}, H_{d}\right)$ are vector-like fields under these $U(1)$ s. Including the soft terms (6.1) economically breaks two of them, and hence $U(1)_{X, Y}$ in Table 5 remain intact. It is important to notice that, in the electroweak broken vacuum (5.1), $U(1)_{X}$ is unbroken and the Nambu-Goldstone boson of $U(1)_{Y}$ is partly absorbed by the massive gauge boson $Z$ and becomes unphysical. Therefore no physical Nambu-Goldstone fields are associated with symmetry breaking. We numerically checked by examining the neutral Higgs mass matrix that there appear no massless scalar bosons. (ii) The second reason is that, when including the breaking terms (6.1), the stationary conditions can still be solved without imposing any tuning of model parameters. (iii) Flavor breaking effects in supersymmetry-breaking holomorphic mass 
terms do not propagate to other sectors. Such a favorable property for the model simplicity does not hold in cases that flavor symmetry violation resides in other supersymmetry-breaking sectors.

The model also predicts a light Higgs boson exactly parallel to the MSSM case. In order to find an approximate form of the Higgs mass spectrum, we take a simplifying assumption:

$$
\begin{gathered}
\left|\mu_{x}\right|^{2}+m_{u x}^{2}=\left|\mu_{x}\right|^{2}+m_{d x}^{2} \equiv \bar{m}^{2}, \quad b_{x} \equiv \bar{b}, \quad(x=S, A, D) \\
b_{S D}=b_{S D}^{\prime}=b_{A D}=b_{A D}^{\prime} \equiv \bar{b}^{\prime}
\end{gathered}
$$

with a hierarchy $\bar{m}^{2},|\bar{b}|,\left|\bar{b}^{\prime}\right| \gg v^{2} \equiv v_{u 2}^{2}+v_{d 1}^{2}+v_{u A}^{2}+v_{d A}^{2}$. In this case, we can analytically write down the mass matrices of Higgs fields. In particular, for the real parts of neutral Higgs bosons, the mass matrix is given by

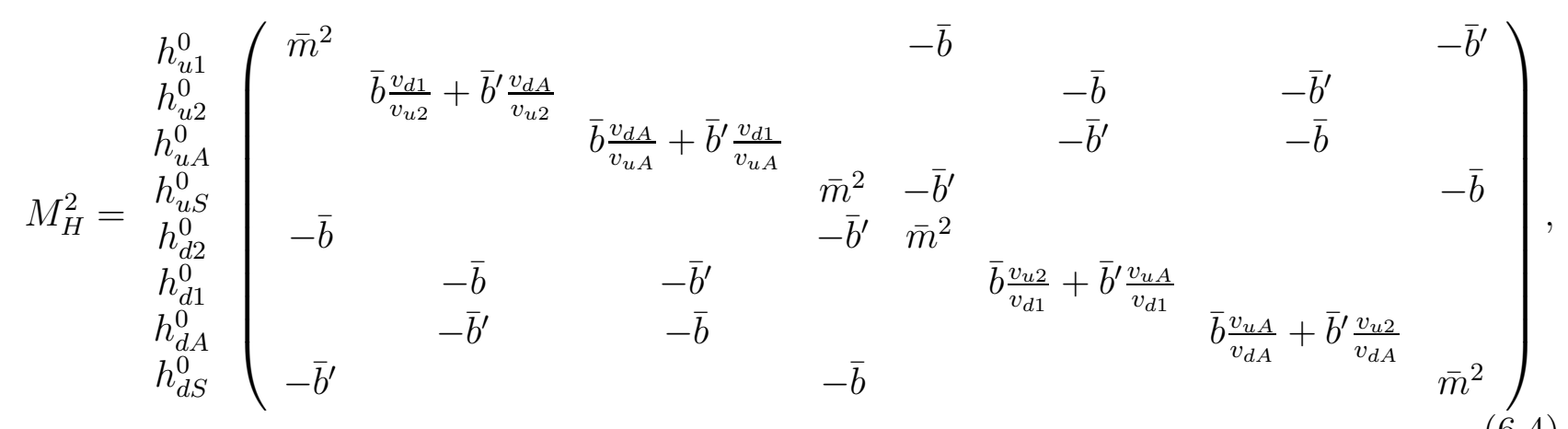

where $h_{x}^{0}(x=u 1, u 2, \cdots)$ are the neutral components of Higgs bosons. Diagonalizing this matrix, we obtain the neutral Higgs masses squared:

$$
\left\{M_{h^{0}}^{2}, M_{H_{1}^{0}}^{2}, M_{H_{2}^{0}}^{2}, M_{H_{3}^{0}}^{2}, M_{H_{4}^{0}}^{2}\right\}=\left\{\mathcal{O}\left(v^{2}\right), \bar{m}^{2}-\bar{b}-\bar{b}^{\prime}, \bar{m}^{2}+\bar{b}-\bar{b}^{\prime}, \bar{m}^{2}-\bar{b}+\bar{b}^{\prime}, \bar{m}^{2}+\bar{b}+\bar{b}^{\prime}\right\},
$$

and the other three mass eigenvalues squared are written in rather complicated expressions, but of the order of $\mathcal{O}\left(\bar{m}^{2}, \bar{b}, \bar{b}^{\prime}\right)$. We therefore have a light mode with a weak scale mass $\mathcal{O}(v)$ and its eigenfunction is explicitly given by

$$
h^{0}=\frac{1}{v}\left(v_{u A} h_{u A}^{0}+v_{d A} h_{d A}^{0}+v_{u 2} h_{u 2}^{0}+v_{d 1} h_{d 1}^{0}\right) .
$$

This light Higgs mode receives a sizable radiative correction from large top Yukawa coupling similar to the MSSM, and would be made heavy enough to satisfy the lower bound from the LEP experiment [22]. 




Figure 1: A Higgs-mediated tree-level FCNC process for the $\mathrm{K}$ meson system.

\section{Tree-level FCNC}

Since there are multiple electroweak doublet Higgses which couple to matter fields, flavorchanging processes are mediated at classical level by these Higgs fields. In the previous section, we show that all but one Higgs bosons have masses of the order of supersymmetry-breaking parameters. Therefore the experimental (un)observations of FCNC rare events would lead to a bound on the supersymmetry breaking scale. Among various experimental constraints, we find the most important constraint comes from the neutral $\mathrm{K}$ meson mixing, which gives a lower bound on heavy Higgs masses being larger than a few TeV.

The down quark mass matrix in Eq. (5.18) is diagonalized as

$$
\begin{gathered}
U_{L}^{\dagger} M_{d} U_{R}=\left(\begin{array}{lll}
m_{d} & & \\
& m_{s} & \\
& & m_{b}
\end{array}\right), \\
U_{L} \simeq\left(\begin{array}{ccc}
1 & \frac{b_{d}}{e_{d}} & 0 \\
-\frac{b_{d}}{e_{d}} & 1 & -\frac{e_{d} d_{d}}{i_{d}^{2}} \\
-\frac{b_{d} d_{d}}{i_{d}^{2}} & \frac{e_{d} d_{d}}{i_{d}^{2}} & 1
\end{array}\right), \quad U_{R} \simeq\left(\begin{array}{ccc}
1 & \frac{d_{d}}{e_{d}} & -\frac{e_{d}}{i_{d}} \\
-\frac{d_{d}}{e_{d}} & 1 & 0 \\
\frac{e_{d}}{i_{d}} & \frac{d_{d}}{i_{d}} & 1
\end{array}\right) .
\end{gathered}
$$

Here and hereafter in this section, we simply take $b_{d} \simeq d_{d}$. The other parameters are fixed by the mass eigenvalues. Tree-level FCNC is mediated by Higgs fields like the diagram shown in Fig. 1. For the heavy mass eigenstates $H_{i}^{0}(i=1,2,3,4)$, the tree-level $K_{L}-K_{S}$ mass difference $\Delta m_{K}^{\text {tree }}$ is given by the matrix element of the effective Hamiltonian between $\mathrm{K}$ mesons [27], 
which is analytically evaluated from the above mixing matrices in the present case as

$$
\begin{aligned}
\Delta m_{K}^{\text {tree }} & =2 \operatorname{Re}\left\langle K^{0}\left|H_{\mathrm{eff}}\right| \bar{K}^{0}\right\rangle \\
& \sim \frac{m_{b}^{2} m_{K} f_{K}^{2}}{6 v_{d 1}^{2} M_{H}^{2}}\left[\left(\frac{m_{K}}{m_{s}+m_{d}}\right)^{2}\left(\frac{m_{s}}{m_{b}}\right)^{2}-\eta^{2}\left\{\left(\frac{m_{K}}{m_{s}+m_{d}}\right)^{2}+\frac{1}{2}\right\}\right],
\end{aligned}
$$

where $m_{K}$ and $f_{K}$ are the mass and decay constant of $\mathrm{K}$ meson and $M_{H}$ is an average of the Higgs masses, $1 / M_{H}^{2}=\frac{1}{4}\left(1 / M_{H_{1}^{0}}^{2}+1 / M_{H_{2}^{0}}^{2}+1 / M_{H_{3}^{0}}^{2}+1 / M_{H_{4}^{0}}^{2}\right)$. The parameter $\eta$ contains the down-type quark Yukawa couplings which do not contribute to mass terms and explicitly given by

$$
\eta=\frac{\left(y_{d}^{S}\right)_{22} b_{d} v_{d 1}}{m_{b}^{2}}-\frac{\left(y_{d}^{S}\right)_{13} d_{d} v_{d 1}}{m_{s} m_{b}},
$$

where $y_{d}^{S}$ denotes the matrix of Yukawa coupling of down-type quarks to the $S_{3}$ singlet Higgs boson. The $S_{3}$ singlet Higgs $H_{S}$ has a vanishing VEV in the present vacuum, and therefore $\eta$ is regarded as a free parameter. For example, $\eta=0$ if one does not include $H_{S}$ in the theory. The other Higgs fields also contribute to the $\mathrm{K}$ meson mixing in a similar order.

In order to estimate the bound on supersymmetry breaking scale, we calculate the ratio of the exact numerical value $\Delta m_{K}^{\text {tree }}$ to the standard contribution $\Delta m_{K}^{\mathrm{SM}}$. In Fig. 2, we show the ratio versus the averaged Higgs mass $M_{H}$ for the cases of $\eta=0$ and $\eta=0.03$ as typical values. It is found from the figure that heavy Higgs masses, in turn the supersymmetry breaking masses, are bounded from below so as to suppress the extra Higgs contribution compared with the standard model one, which bound is roughly given by

$$
M_{H} \geq\left\{\begin{array}{ll}
3.8 \mathrm{TeV} & (\eta=0) \\
1.4 \mathrm{TeV} & (\eta=0.03)
\end{array},\right.
$$

where we have used the experimental data $m_{K}=490 \mathrm{MeV}, f_{K}=160 \mathrm{MeV}$ and taken $v_{d 1}=$ $100 \mathrm{GeV}$ as a typical electroweak scale. Other flavor-changing rare processes are also suppressed for such heavy Higgs fields of few TeVs. For example, the $\mu \rightarrow e+\gamma$ process is given in terms of extra Higgs masses and lepton Yukawa couplings [28]. The branching ratio is found to be suppressed enough under the constraint of eq.(7.5). 


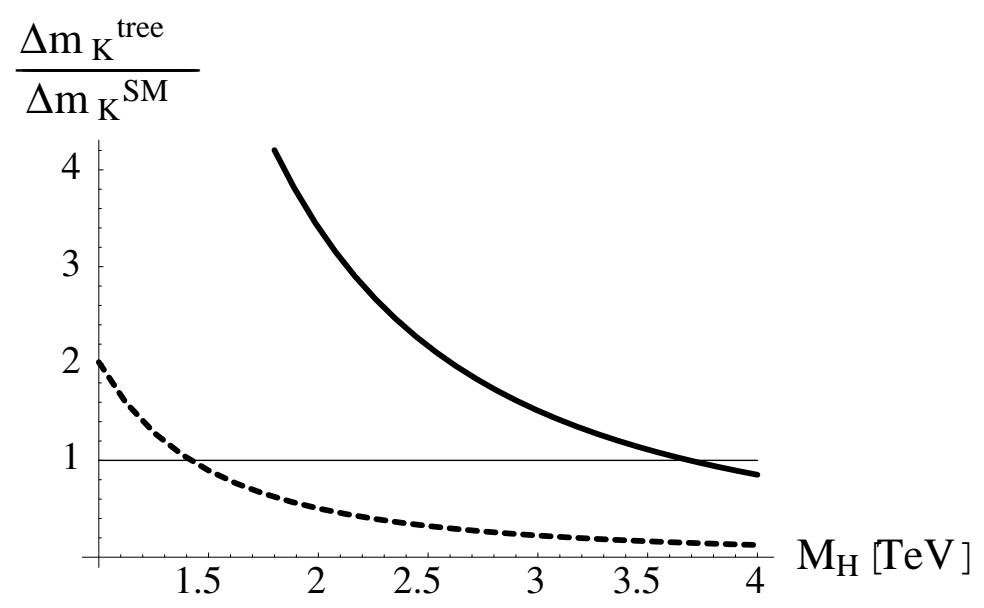

Figure 2: $\Delta m_{K}^{\text {tree }} / \Delta m_{K}^{\mathrm{SM}}$ as the function of Higgs mass parameter $M_{H}$. The solid and dashed lines correspond to $\eta=0$ and 0.03 , respectively.

\section{Summary}

In this paper we have discussed the structure of Higgs potential and fermion mass matrices in supersymmetric models with $S_{3}$ flavor symmetry. The electroweak doublet Higgs fields belong to non-trivial representations of the flavor symmetry and lead to restricted forms of vacuum structure. We have examined possible zero elements (textures) of quark and lepton mass matrices. Our approach to have mass textures is rather different from previous ones in a sense that flavor symmetry does not forbid coupling constants of (effective) Yukawa operators, but leads to mass texture zeros by ensuring some of Higgs fields have vanishing expectation values in the vacuum of the theory. The vanishing mass matrix elements are obtained dynamically in the vacuum without any exact tuning of model parameters, and their positions are controlled by flavor symmetry. An interesting point is that, due to the flavor group structure, the up and down quark mass matrices are automatically made different, which lead to non-vanishing generation mixing. We have exhausted the patterns of flavor symmetry charges of matter fields and found that the simplest, viable forms of mass matrices including the neutrino sector are uniquely determined. In particular the lepton mixing $V_{e 3}$ is predicted within the range that will be tested in near future experiments.

We have also discussed the physical mass spectrum of Higgs bosons and its phenomeno- 
logical implication. The flavor symmetry is softly broken by a certain class of holomorphic supersymmetry-breaking mass terms of Higgs bosons. That neither destabilizes the desired vacuum nor introduces any fine tuning of coupling constants. Given these flavor breaking terms, it is found that all Higgs bosons, except for the lightest one, can be made heavier than a few TeV enough to satisfy the experimental bounds such as that from the neutral $\mathrm{K}$ meson mixing. Detail phenomenological analyses including CP violation and FCNC will be presented elsewhere.

We would like to thank J. Kubo, T. Kobayashi and H. Nakano for helpful discussions. This work was supported in part by scientific grants from the Ministry of Education, Science, Sports, and Culture of Japan (No. 17540243 and 17740150), and grant-in-aid for the scientific research on priority area (\#441) "Progress in elementary particle physics of the 21st century through discoveries of Higgs boson and supersymmetry" (No. 16081209). 


\section{References}

[1] S. Pakvasa and H. Sugawara, Phys. Lett. B 73 (1978) 61;

D. Wyler, Phys. Rev. D 19 (1979) 330;

L.J. Hall and H. Murayama, Phys. Rev. Lett. 75 (1995) 3985;

R. Dermisek and S. Raby, Phys. Rev. D 62 (2000) 015007;

R.N. Mohapatra, A. Perez-Lorenzana and C.A. de Sousa Pires, Phys. Lett. B 474 (2000) 355;

J. Kubo, A. Mondragon, M. Mondragon and E. Rodriguez-Jauregui, Prog. Theor. Phys. 109 (2003) 795 [Erratum-ibid. 114 (2005) 287];

T. Kobayashi, J. Kubo and H. Terao, Phys. Lett. B 568 (2003) 83;

P.F. Harrison and W.G. Scott, Phys. Lett. B 557 (2003) 76;

J. Kubo, H. Okada and F. Sakamaki, Phys. Rev. D 70 (2004) 036007;

S.-L. Chen, M. Frigerio and E. Ma, Phys. Rev. D 70 (2004) 073008 [Erratum-ibid. D 70 (2004) 079905];

W. Grimus and L. Lavoura, JHEP 0508 (2005) 013;

Y. Koide, Phys. Rev. D 73 (2006) 057901.

[2] N. Haba and K. Yoshioka, Nucl. Phys. B 739 (2006) 254.

[3] Y. Yamanaka, H. Sugawara and S. Pakvasa, Phys. Rev. D 25 (1982) 1895 [Erratum-ibid. D 29 (1984) 2135];

C. Hagedorn, M. Lindner and R. N. Mohapatra, JHEP 0606 (2006) 042.

[4] W. Grimus and L. Lavoura, Phys. Lett. B 572 (2003) 189;

W. Grimus, A. S. Joshipura, S. Kaneko, L. Lavoura and M. Tanimoto, JHEP 0407 (2004) 078 ;

W. Grimus, A. S. Joshipura, S. Kaneko, L. Lavoura, H. Sawanaka and M. Tanimoto, Nucl. Phys. B 713 (2005) 151. 
[5] K.S. Babu and J. Kubo, Phys. Rev. D 71 (2005) 056006;

Y. Kajiyama, E. Itou and J. Kubo, Nucl. Phys. B 743 (2006) 74.

[6] M. Frigerio, S. Kaneko, E. Ma and M. Tanimoto, Phys. Rev. D 71 (2005) 011901.

[7] E. Ma and G. Rajasekaran, Phys. Rev. D 64 (2001) 113012;

E. Ma, Mod. Phys. Lett. A 17 (2002) 2361;

K.S. Babu, E. Ma and J.W.F. Valle, Phys. Lett. B 552 (2003) 207;

M. Hirsch, J.C. Romao, S. Skadhauge, J.W.F. Valle and A. Villanova del Moral, Phys. Rev. D 69 (2004) 093006;
A. Zee, Phys. Lett. B 630 (2005) 58;
G. Altarelli and F. Feruglio, Nucl. Phys. B 720 (2005) 64;
X.-G. He, Y.-Y. Keum and R.R. Volkas, JHEP 0604 (2006) 039;
E. Ma, H. Sawanaka and M. Tanimoto, hep-ph/0606103.

[8] E. Derman and H.S. Tsao, Phys. Rev. D 20 (1979) 1207;

D. Chang, W.Y. Keung and G. Senjanovic, Phys. Rev. D 42 (1990) 1599;

D.B. Kaplan and M. Schmaltz, Phys. Rev. D 49 (1994) 3741;

P.H. Frampton and T.W. Kephart, Int. J. Mod. Phys. A 10 (1995) 4689;

A. Aranda, C.D. Carone and R.F. Lebed, Phys. Lett. B 474 (2000) 170;

N. Haba, A. Watanabe and K. Yoshioka, Phys. Rev. Lett. 97 (2006) 041601;

C. Hagedorn, M. Lindner and F. Plentinger, Phys. Rev. D 74 (2006) 025007.

[9] H. Fritzsch, Phys. Lett. B 73 (1978) 317;

P. Ramond, R.G. Roberts and G.G. Ross, Nucl. Phys. B 406 (1993) 19.

[10] H. Nishiura, K. Matsuda and T. Fukuyama, Phys. Rev. D 60 (1999) 013006;

E.K. Akhmedov, G.C. Branco and M.N. Rebelo, Phys. Rev. Lett. 84 (2000) 3535;

S.K. Kang and C.S. Kim, Phys. Rev. D 63 (2001) 113010. 
[11] P.H. Frampton, S.L. Glashow and D. Marfatia, Phys. Lett. B 536 (2002) 79;

Z.-z. Xing, Phys. Lett. B 530 (2002) 159;

R. Barbieri, T. Hambye and A. Romanino, JHEP 0303 (2003) 017;

A. Ibarra and G.G. Ross, Phys. Lett. B 575 (2003) 279.

[12] A. Kageyama, S. Kaneko, N. Shimoyama and M. Tanimoto, Phys. Lett. B 538 (2002) 96;

S. Kaneko and M. Tanimoto, Phys. Lett. B 551 (2003) 127;

M. Honda, S. Kaneko and M. Tanimoto, JHEP 0309 (2003) 028; Phys. Lett. B 593 (2004) 165 ;

S. Kaneko, H. Sawanaka and M. Tanimoto, JHEP 0508 (2005) 073.

[13] M.-C. Chen and K.T. Mahanthappa, Phys. Rev. D 68 (2003) 017301;

M. Bando and M. Obara, Prog. Theor. Phys. 109 (2003) 995;

M. Bando, S. Kaneko, M. Obara and M. Tanimoto, Phys. Lett. B 580 (2004) 229.

[14] L. Lavoura, Phys. Lett. B 609 (2005) 317;

W. Grimus, S. Kaneko, L. Lavoura, H. Sawanaka and M. Tanimoto, JHEP 0601 (2006) 110.

[15] M. Frigerio and A. Y. Smirnov, Phys. Rev. D 67 (2003) 013007.

[16] N. Uekusa, A. Watanabe and K. Yoshioka, Phys. Rev. D 71 (2005) 094024;

A. Watanabe and K. Yoshioka, JHEP 0605 (2006) 044.

[17] C. Hagedorn and W. Rodejohann, JHEP 0507 (2005) 034;

A. Merle and W. Rodejohann, Phys. Rev. D 73 (2006) 073012.

[18] M. Dine, Y. Nir and Y. Shirman, Phys. Rev. D 55 (1997) 1501;

R. Rattazzi an U. Sarid, Nucl. Phys. B 501 (1997) 297;

M. Yamaguchi and K. Yoshioka, Phys. Lett. B 543 (2002) 189.

[19] J.P. Derendinger, J.E. Kim and D.V. Nanopoulos, Phys. Lett. B 139 (1984) 170;

I. Antoniadis, J.R. Ellis, J.S. Hagelin and D.V. Nanopoulos, Phys. Lett. B 194 (1987) 231. 
[20] H. Georgi and S.L. Glashow, Phys. Rev. Lett. 32 (1974) 438.

[21] K.S. Babu and Q. Shafi, Phys. Rev. D 47 (1993) 11;

K.S. Babu and R.N. Mohapatra, Phys. Rev. Lett. 74 (1995) 2418.

[22] S. Eidelman et al. [Particle Data Group Collaboration], Phys. Lett. B 592 (2004) 1.

[23] H. Georgi and C. Jarlskog, Phys. Lett. B 86 (1979) 297;

S. Dimopoulos, L.J. Hall and S. Raby, Phys. Rev. Lett. 68 (1992) 1984.

[24] Z. Maki, M. Nakagawa and S. Sakata, Prog. Theor. Phys. 28 (1962) 870.

[25] T. Yanagida, in Proceedings of the "Workshop on the Unified Theory and the Baryon Number in the Universe”, Tsukuba, Japan, 1979, edited by O. Sawada and A. Sugamoto, KEK Report No. KEK-79-18, p. 95; Prog. Theor. Phys. 64 (1980) 1103;

P. Ramond, in Talk given at the Sanibel Symposium, Palm Coast, Fla., 1979, preprint CALT-68-709;

S.L. Glashow, in Quarks and leptons, proceedings of the advanced study institute (Cargèse, Corsica, 1979), J.-L. Basdevant et al. eds., Plenum, New York 1981;

for an early work, see P. Minkowski, Phys. Lett. B 67 (1977) 421;

See also R.N. Mohapatra and G. Senjanovic, Phys. Rev. Lett. 44 (1980) 912.

[26] K. Anderson et al., "White paper report on using nuclear reactors to search for a value of $\theta_{13} "$, hep-ex/0402041.

[27] F. Gabbiani, E. Gabrielli, A. Masiero and L. Silvestrini, Nucl. Phys. B 477 (1996) 321.

[28] For example, E. O. Iltaqn, JHEP 0402 (2004) 065. 